\title{
Tubulointerstitial Injury Induced in Rats by a Monoclonal Antibody That Inhibits Function of a Membrane Inhibitor of Complement
}

\author{
Atsushi Nomura, * Kazuhiro Nishikawa, “ Yukio Yuzawa, * Hidechika Okada, ${ }^{\S}$ Noriko Okada, ${ }^{\S}$ B. Paul Morgan," \\ Sara J. Piddlesden," Masayuki Nadai, ${ }^{\ddagger}$ Takaaki Hasegawa, ${ }^{\ddagger}$ and Seiichi Matsuo* \\ *Third Department of Internal Medicine and ${ }^{\ddagger}$ Department of Hospital Pharmacy, Nagoya University School of Medicine, Nagoya 466, \\ Japan; ${ }^{\S}$ Department of Molecular Biology, Nagoya City University School of Medicine, Nagoya 467, Japan; and "Department of Medical \\ Biochemistry, University of Wales College of Medicine, Cardiff CF4 4XN, United Kingdom
}

\begin{abstract}
The kidney widely expresses membrane-associated complement regulatory proteins (membrane inhibitors of complement). The aim of this work was to evaluate the roles of these molecules in rat kidneys in vivo. To suppress functions of rat membrane inhibitors of complement, two mAbs, $5 \mathrm{I} 2$ and 6D1, were used. 5I2 and 6D1 inhibit functions of membrane inhibitors of complement at $\mathrm{C3}$ level (rat $\mathrm{Crry} / \mathrm{p65}$ ) and C8/9 level (rat CD59), respectively. F(ab')2 fragment of 512 or $6 \mathrm{D1}$ was perfused in the left kidneys, and perfusate was discarded from the renal vein. After perfusion, the left kidneys were connected to systemic circulation. In rats perfused with 5I2, mouse IgG was found in glomeruli, peritubular capillaries, vascular bundles, and tubules 15 min after recirculation. Binding of $\mathrm{C} 3$ and $\mathrm{C} 5 \mathrm{~b}-9$ was evident in these areas. $1 \mathrm{~d}$ after perfusion with 5I2, cast formation, dilatation of tubular lumen, and tubular cell degeneration were observed. At day 4 through day 7 , significant mononuclear cell infiltration and proximal tubule damage were observed. These changes were completely prevented by complement depletion. Rats perfused with 6D1 showed the binding of mouse IgG in the similar areas as $5 \mathrm{I} 2$, but $\mathrm{C3}$ or $\mathrm{C5b}-9$ deposition was not observed. Rats perfused with 6D1 or vehicle only did not show any pathology in the left kidneys.

These results suggest that rat Crry/p65 plays protective roles against spontaneously occurring indiscriminate attack to tubulointerstitial tissues by autologous complement and that rat Crry/p65 is one of the important factors to maintain normal integrity of the kidney in rats. (J. Clin. Invest. 1995. 96:2348-2356.) Key words: alternative pathway • tubulointerstitial nephritis • 5I2Ag • Crry/p65 • CD59
\end{abstract}

\section{Introduction}

Tubulointerstitial injury is an important determinant of kidney function because it correlates better with glomerular filtration

Part of this work was presented at the 27th annual meeting of the American Society of Nephrology (26-29 October 1994, Orlando, FL) and was published in abstract form (1994. J. Am. Soc. Nephrol. 5:760a).

Address correspondence to Seiichi Matsuo, The Third Department of Internal Medicine, Nagoya University School of Medicine, 65 Tsuruma-cho, Showa-ku, Nagoya 466, Japan. Phone: 81-52-741-2111, Ext. 2219; FAX: 81-52-782-0922.

Received for publication 6 February 1995 and accepted in revised form 12 July 1995.

J. Clin. Invest.

(C) The American Society for Clinical Investigation, Inc. 0021-9738/95/11/2348/09 \$2.00

Volume 96, November 1995, 2348-2356 rate than glomerular injury $(1,2)$. Several immunological and nonimmunological mechanisms of progressive tubulointerstitial injury have been proposed (3) including autologous complement activation in the kidney parenchyma $(4,5)$. Ammonia is thought to be a major nonimmunological activator of alternative pathway of complement in the kidney $(6,7)$. In the normal condition, however, complement activation is well regulated in the kidney, and normal renal function is maintained. We have suggested that cell membrane-associated complement regulatory proteins (membrane inhibitors of complement) expressed abundantly in the kidney play protective roles against complement-mediated renal injuries (8).

To assess in vivo roles of membrane inhibitors of complement, we have used neutralizing mAbs against rat inhibitors. Our recent study revealed that the systemic administration of mAb 5I2 (9) which inhibits functions of a rat membrane inhibitor at C3 level (5I2Ag [10] or a rat counterpart of mouse Crry/ p65 [11-14]), induced deposition of C3 in the capillary endothelial cells resulting in symptoms resembling endotoxin shock in rats (decreased blood pressure, increased vascular permeability, and leukocyte infiltration in organs) (15). In contrast, administration of mAb 6D1 (16), which neutralizes functions of rat CD59, did not induce significant change although 6D1 bound to the capillary endothelial cells in a pattern similar to $5 \mathrm{I} 2$ (15). The findings obtained in our previous work prompted us to investigate the consequences of in vivo suppression of membrane inhibitors of complement in the kidney by specific mAbs.

Results obtained in the present work demonstrated for the first time that in vivo suppression by a specific $\mathrm{mAb}$ of a renal membrane inhibitor operating at $\mathrm{C} 3$ but not $\mathrm{C} 8 / 9$ level induced complement-dependent tubulointerstitial injury in rats and provided supporting evidence that complement system is potentially harmful in the normal kidney if membrane inhibitors of complement fail to play protective roles against indiscriminate attack of autologous complement. The details of experiments and the possible relevance to progressive tubulointerstitial injury will be discussed.

\section{Methods}

Animals. Female Wistar rats weighing $\sim 280 \mathrm{~g}$ were purchased from Chubu Kagaku Shizai Co. Ltd. (Nagoya, Japan) and were allowed free access to food and water.

Antibodies. Characteristics of rat Crry/p65 (5I2Ag) and mouse mAb $5 \mathrm{I} 2$ were described previously $(9,10) . \mathrm{F}\left(\mathrm{ab}^{\prime}\right) 2$ fragment was prepared as reported earlier (15). A mouse mAb 6D1 (16) that inhibits function of rat CD59 (17) was described previously. Mouse mAb 2A1 (18) directed against rat C5b-9 was kindly provided by Dr. W. G. Couser (University of Washington, Seattle, WA). 2Al was labeled with biotin according to the method described by Guesdon et al (19). Cobra venom 
Table I. Experimental Protocol

\begin{tabular}{rlccccc}
\hline Group & Antibody & CVF & 15 min & Day 1 & Day 4 & Day 7 \\
\hline I & 512 F(ab)'2 & - & 3 & 6 & 7 & 6 \\
II & $512 \mathrm{~F}(\mathrm{ab})^{\prime} 2$ & + & 3 & 6 & 6 & 6 \\
III & vehicle & - & 3 & 6 & 6 & 6 \\
IV & 6D1 F(ab)'2 & - & 3 & 6 & 6 & 0
\end{tabular}

The number in each column indicates the number of rats examined. Vehicle, modified Tyrode's buffer.

factor $(\mathrm{CVF})^{1}$ was routinely purified as described before (15). FITClabeled mouse mAb against rat leukocyte common antigen (LCA) (clone OX-1) was purchased from Dainippon Pharmaceutical Co. (Osaka, Japan). An mAb against vimentin (clone V9) was purchased from Dako Corp. (Santa Barbara, CA) and biotinylated as described above.

Serum CH50. Blood samples were drawn from the tail vein into syringes containing EDTA (Sigma Chemical Co., St. Louis, MO) before, $3 \mathrm{~h}, 24 \mathrm{~h}$, and $4 \mathrm{~d}$ after kidney perfusion. Serum CH50 concentration was measured using sensitized sheep red blood cells (Ishizu Pharmaceutical Co., Osaka, Japan) according to manufacturer's direction.

Urinary protein excretion. Rats were housed in metabolic cages overnight $(16 \mathrm{~h})$ everyday from day 0 to day 5 . Urinary protein was measured by pyrogallol red method (20).

Kidney perfusion. To localize $5 \mathrm{I} 2$ solely in the left kidney and to avoid the systemic effects of 5I2, isolated left kidney perfusion was performed according to the method described previously $(21,22)$. All the perfusate was discarded through the cannula in the vein. The kidney was perfused at a rate of $2 \mathrm{ml} / \mathrm{min}$ using a peristaltic pump. After kidney perfusion, tubes were removed, and the holes of the vessels were repaired by microsurgery. Then blood circulation was reestablished by releasing the clamps. The average time required for whole procedure was $\sim 15 \mathrm{~min}$

Experimental protocol. Rats were divided into four groups. Rats of group I were perfused with $0.3 \mathrm{mg}$ of $\mathrm{F}\left(\mathrm{ab}^{\prime}\right) 2$ fragment of 512 in 10 $\mathrm{ml}$ of Tyrode's buffer. Rats of group II were depleted of complement by intravenous injection of $25 \mathrm{U}$ of purified CVF. After $24 \mathrm{~h}$, kidneys were perfused with $0.3 \mathrm{mg}$ of $\mathrm{F}\left(\mathrm{ab}^{\prime}\right) 2$ fragment of 512. Group III rats were perfused with Tyrode's buffer (vehicle) $(21,22)$ only and used as controls. Group IV rats were perfused with $0.3 \mathrm{mg}$ of $F\left(a b^{\prime}\right) 2$ fragment of $6 \mathrm{D} 1$. Rats were killed at 1,4 , and $7 \mathrm{~d}$ after perfusion. To see the binding of mAbs and rat complement, rats were also killed 15 min after perfusion. Protocol and the number of rats examined are summarized in Table I.

Histology and immunohistology. For light microscopic examination, methacarn-fixed and paraffin-embedded kidney tissues were cut at $2 \mu \mathrm{m}$ and stained with periodic acid-Schiff (PAS). To assess tubulointerstitial injuries, kidney sections were arbitrary divided into three regions, i.e., cortex, outer medulla, and inner medulla. Using semiquantitative indices, sections were analyzed for the evaluation of acute tubulointerstitial damage. In each region, extents of tubular cast formation, tubular dilatation, and tubular degeneration (vacuolar change, loss of brush border, detachment of tubular epithelial cells, and condensation of tubular nuclei) were scored according to the following criteria by two blinded observers: 0 , normal; $1,<30 \% ; 2,30 \%-70 \% ; 3,>70 \%$ of the pertinent area.

For immunofluorescence microscopy, part of the kidney tissues were snap frozen, cut at $2 \mu \mathrm{m}$, and fixed in acetone. Sections were stained

1. Abbreviations used in this paper: CVF, cobra venom factor; LCA, leukocyte common antigen; PAH, para-aminohippurate; RPF, renal plasma flow. by FITC-labeled rabbit antibodies against mouse IgG and rat IgG, and FITC-labeled goat antibodies against rat C3 (Cappel Laboratories, West Chester, PA). For the detection of C5b-9 and vimentin, sections were incubated with biotinylated $2 \mathrm{~A} 1$ and V9 after treatment with avidin D and biotin-blocking solution (Vector Laboratories, Burlingame, CA) and then incubated with FITC-labeled streptoavidin (Cappel Laboratories). Deposition of rat C3 and C5b-9 in the kidney was semiquantitively scored according to the extent of deposition in each region using the following criteria: 0 , normal; $1,<30 \% ; 2,30 \%-70 \%$; and $3,>70 \%$ of the pertinent area. Since normal rat tubular basement membrane of cortex was positively stained for rat $\mathrm{C} 3$ and $\mathrm{C} 5 \mathrm{~b}-9$ in a focal and interrupted pattern, only circumferential staining was counted as positive. In renal medulla, C3 and C5b-9 were negative in normal rats. Proximal tubular cell damage was evaluated by the staining for vimentin intermediate filaments $(23,24)$. Vimentin was assessed only in the renal cortex according to the extent of vimentin staining in the proximal tubules using the same criteria as for rat $\mathrm{C} 3$ and $\mathrm{C} 5 \mathrm{~b}-9$. For analysis of interstitial leukocyte infiltration, sections were stained by FITC-OX1. The number of positive cells was counted in randomly selected 10 microscopic fields under higher magnification $(\times 400)$. After washing with PBS, all the sections were covered with $90 \%$ glycerol containing $p$-phenylenediamine (25) and were examined by two blinded observers using an epifluorescence microscope (Olympus Optical Co., Tokyo, Japan).

Renal function study. In a separate set of experiment, studies for renal function of the perfused kidneys were performed. 24 rats were divided into four groups (groups I-IV) according to the protocol described above. All rats underwent studies for renal function at day 4 . Polyethylene tubes were placed into right jugular vein, left internal carotid artery, and left ureter under pentobarbital anesthesia. To determine inulin and para-aminohippurate (PAH) clearance, solution of saline-containing inulin $(0.57 \mathrm{mg} / \mathrm{min})$ and PAH $(0.29 \mathrm{mg} / \mathrm{min})$ was infused from venous catheter after a priming dose of $100 \mathrm{mg} / \mathrm{kg}$ body wt inulin. After 30 min period for equilibration, urine and blood samples were collected from left ureter and carotid artery. After the clearance study, blood samples were collected from arterial catheter and by left renal vein puncture simultaneously, and the renal extraction of PAH was calculated. Plasma and urine concentration of inulin and PAH was measured by the colorimetric methods described by Dische et al. (26) and Marshall (27), respectively. GFR was determined from inulin clearance, and renal plasma flow (RPF) was calculated from PAH clearance and extraction.

Statistical analysis. All values are provided as mean \pm SE. Statistical analysis was performed by one-factor ANOVA. When significant difference was present, statistical analysis was further performed using Scheffe's $F$ test between two groups. Significant difference was set when $P$ value was $<0.05(5 \%)$.

\section{Results}

\section{Light microscopic findings}

Significant tubulointerstitial change was observed by light microscopy in rats of group I. There were significant cast formation and dilatation of tubules along with vacuolar degeneration, numerous mitotic figures, loss of brush border, and detachment of tubular epithelial cells at days 1 and 4 (Fig. 1). Cellular infiltration was prominent at days 4 and 7 (quantitative data are shown in the next section Immunohistological findings). Semiquantitative analysis revealed that the extents of tubular cast formation, dilatation of tubules, and tubular degeneration were significantly larger in group I rats than in other groups of rats (Fig. 2). The tubulointerstitial changes observed by light microscopy were prominent at days $1-4$, and tended to heal by day 7. In rats of group III (controls), there was no histological alteration in left kidneys throughout the experiments. In rats of groups II and IV, there was no significant tubulointerstitial change as compared to the controls (Figs. 1 and 2). 

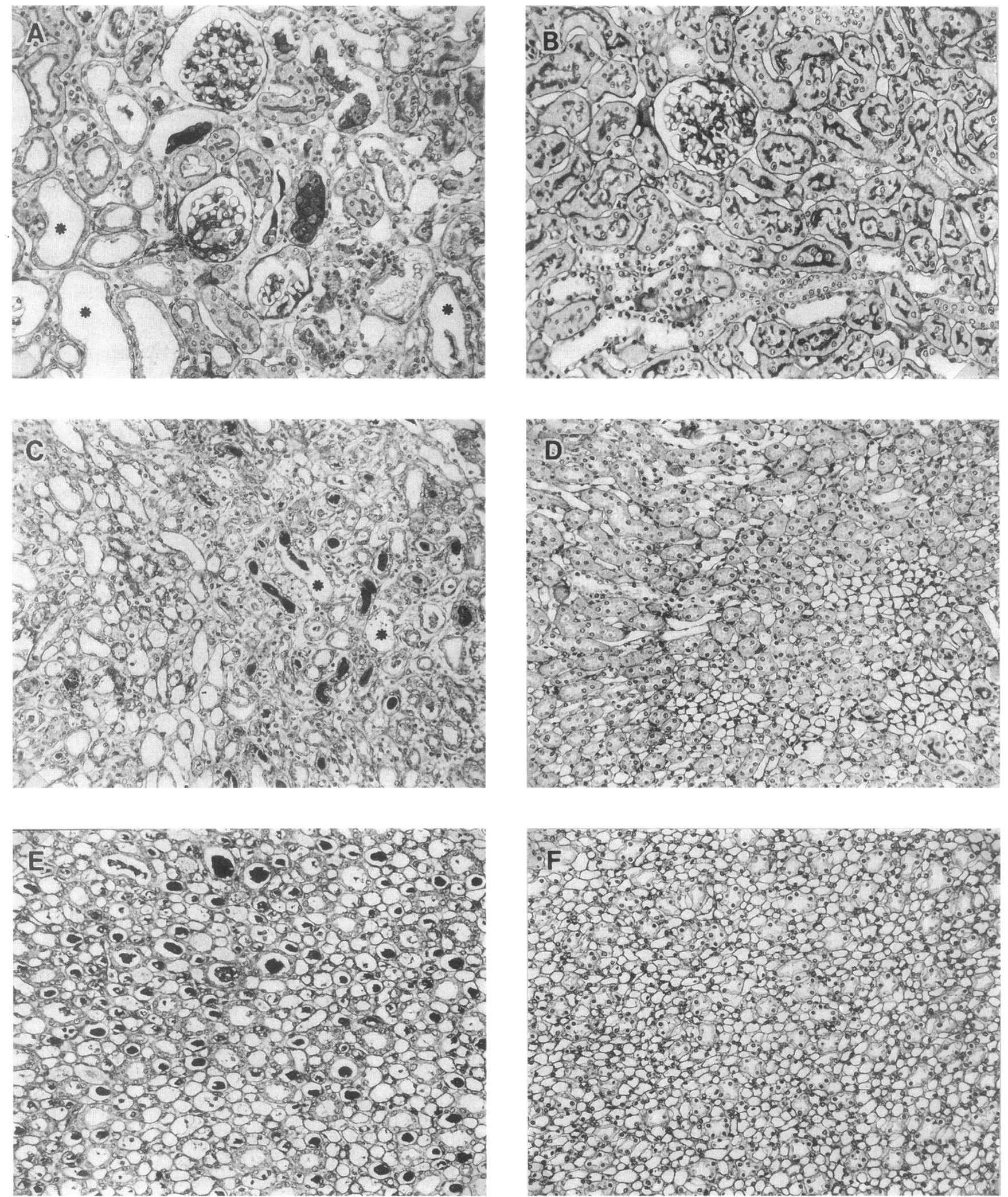

Figure 1. Light microscopic pictures at day 4 in groups I and IV rats. $(A, C$, and $E)$ group I; $(B, D$, and $F)$ group IV; $(A$ and $B)$ cortex (PAS, $\times 200)$; $(C$ and $D$ ) outer medulla (PAS, $\times 200) ;(E$ and $F)$ inner medulla (PAS, $\times 200)$. Tubular dilatation was seen in the cortex and outer medulla in rats of group I ( $A$ and $C$, asterisks). Cellular infiltration is prominent in the outer medulla resulting in the loss of normal architecture $(C)$. In the inner medulla, cast formation and tubular degeneration were seen in rats of group $I(E)$. In contrast, renal structure was normal in rats of group IV $(B, D$, and $F)$. 


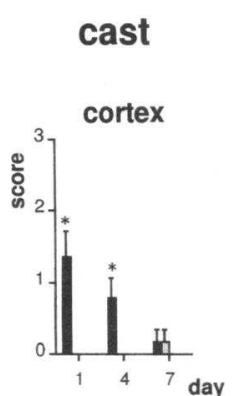

\section{dilatation}

degeneration
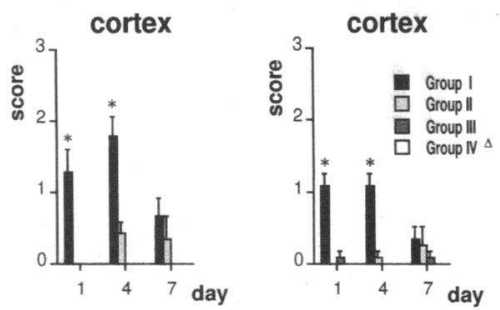

outer medulla

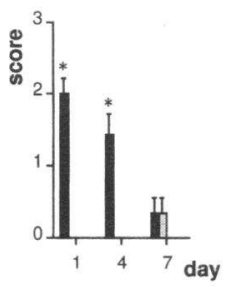

outer medulla
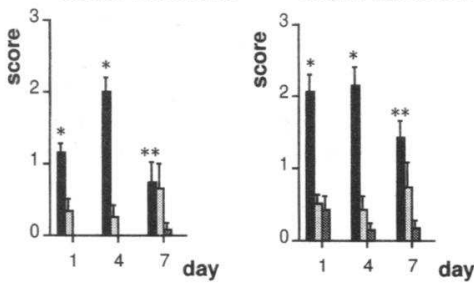

inner medulla
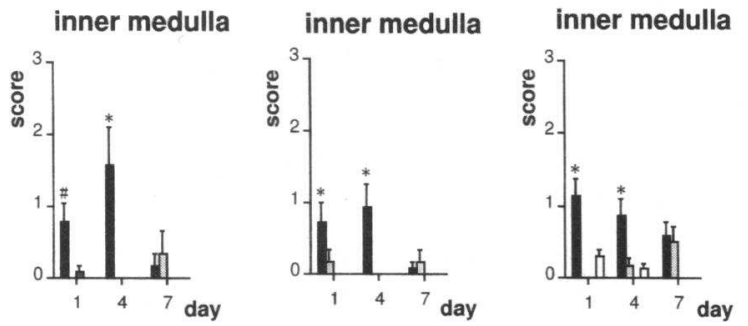

Figure 2. Semiquantitative analysis of tubulointerstitial damage (tubular cast formation, tubular dilatation, and tubular degeneration) using light microscopic specimens. Data are shown as mean (column) and standard error (bar). * Significant difference between group I and other groups; ** significant difference between groups I and III; " significant difference between groups I and II or groups I and IV; ${ }^{\Delta}$ group IV was not examined at day 7 .

In contrast to tubulointerstitial tissue, there was no significant alteration in the glomerulus throughout the experiments in rats of group I and other groups.

\section{Immunohistological findings}

Distribution of rat Crry/p65 in the normal rat kidney. Rat Crry/ p65 is present in the glomerulus (mesangial area and in the capillary walls), tubular basolateral membrane, and/or cytoplasm of tubules, peritubular capillaries, vascular bundles, and in the medullary capillaries as reported previously (28).

Distribution of antibody in vivo. $15 \mathrm{~min}$ after kidney perfusion, binding of mAb 512 was seen along glomerular, peritubular, and medullary capillaries including vascular bundles of outer medulla in rats of groups I (Fig. 3, $A, C$, and $E$ ) and II. There was also antibody binding to the basal membrane of the proximal tubules (Fig. $3 \mathrm{~A}$ ). At day 1 ( $24 \mathrm{~h}$ after perfusion), mouse IgG was still significantly observed in the tubules and vascular bundles while it became nearly undetectable in glomeruli and peritubular capillaries. After $4 \mathrm{~d}$, mouse IgG disappeared from the kidney except for weak staining in vascular bundles. In rats of group IV, 6D1 bound to glomerulus, peritubular capillaries, vascular bundles, and distal tubules $15 \mathrm{~min}$ after perfusion (Fig. 3, $B, D$, and $E$ ). The fate of 6D1 in the kidney was almost identical with that of 512. Rat IgG was not detectable in all rats throughout the experiment.
Deposition of complement. $15 \mathrm{~min}$ after perfusion/recirculation, kidneys from Group I revealed strong deposition of $\mathrm{C} 3$ in peritubular capillaries of cortex, vascular bundles, distal tubules of outer medulla, and in the inner medulla (Fig. 4, $A, C$, and $E)$. C5b-9 was also detected in the same area but the staining intensity was weaker than C3. Complement deposition was only weakly seen in the glomerulus at this early stage. At days 1 and 4, circumferential binding of $\mathrm{C} 3$ and C5b-9 was observed along cortical tubular basement membrane. There was also binding of C3 in the outer and inner medulla. C5b-9 was detected in the tubules and tubular casts. In the glomerulus, complement became no longer detectable at day 1 and onwards. In rats of groups II, III, and IV, there was no abnormal deposition of complement in the kidney (Fig. $4 B, D$, and $F$ ). These rats showed deposition of C3 and C5b-9 only faintly in the vascular bundles and vessels of the inner medulla throughout the experiment. These data were semiquantitated and given in Fig. 5.

Cellular infiltration. In rats of group I, cellular infiltration was not so prominent at day 1 in cortex and medulla. There was, however, significant leukocyte infiltration at days 4 and 7 . Cellular infiltration was most prominent in the outer medulla. In other groups of rats, cellular infiltration was minimal and did not change throughout the experiments. Leukocyte infiltration was not seen in the glomeruli of all rats throughout the experiments. These results are shown in Fig. 6.

Vimentin staining. In normal rats, glomeruli and renal vasculatures are reported to express vimentin intermediate filaments while tubular cells do not express vimentin (23). Distribution of vimentin in the left kidneys of groups II, III, and IV was quite similar to that reported in the normal kidneys. In contrast, significant numbers of tubular epithelial cells of group I rats expressed vimentin from days $4-7$.

\section{Renal function}

GFR and RPF in rats of Group I were not significantly different from rats of other groups at day 4 (Table II).

\section{Serum complement activity}

In rats of groups I, III, and IV, there was no significant change of serum complement activity ( $\mathrm{CH} 50)$ at $3 \mathrm{~h}, 1 \mathrm{~d}$, and $4 \mathrm{~d}$ after perfusion or injection. In group II rats that were intravenously injected with CVF $24 \mathrm{~h}$ before perfusion, CH50 was undetectable at $3 \mathrm{~h}$ and $1 \mathrm{~d}$ after perfusion and returned to the normal level at day 4.

\section{Urinary protein excretion}

Urinary protein significantly increased in rats of group I compared with other groups of rats at days $0-1$. Urinary protein excretion readily came to the normal range at days $2-3$ and onwards. The degree of proteinuria in group I rats was mild $(<15 \mathrm{mg} / 16 \mathrm{~h})($ Fig. 7$)$.

\section{Discussion}

In the normal human kidney, expression of three major membrane inhibitors of complement, membrane cofactor protein (CD46) (29), decay accelerating factor (CD55) (30) and 20$\mathrm{kD}$ homologous restriction factor (HRF20 [31], membrane inhibitor of reactive lysis [32], P18 [33], or CD59 [34, 35]), is observed (8). In the rat kidney, the patterns of distribution of 

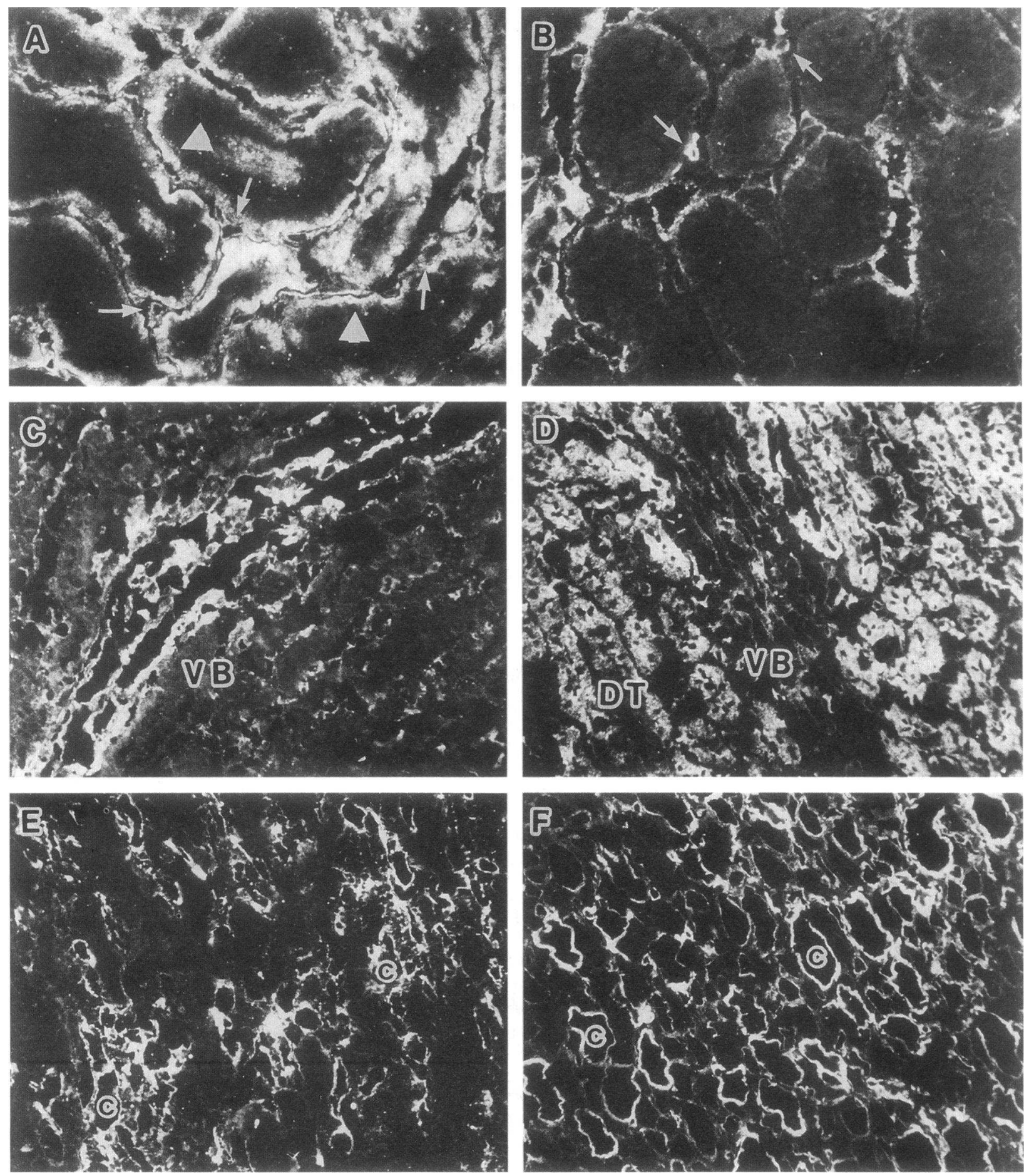

Figure 3. Distribution of mAbs $15 \mathrm{~min}$ after perfusion/recirculation. ( $A, C$, and $E$ ) Group I (5I2); $(B, D$, and $F)$ group IV (6D1); ( $\times 200) ;(A$ and $B)$ cortex; $(C$ and $D)$ outer medulla; $(E$ and $F)$ inner medulla. The kidney perfused with 5 I2 showed binding of mAb in peritubular capillaries (arrows), basal membrane of proximal tubules (arrow heads), vascular bundles (VB), and in the medullary capillaries and collecting ducts (c). The kidney perfused with $6 \mathrm{D} 1$ also showed binding of $\mathrm{mAb}$ in the peritubular capillaries (arrows), distal tubules $(D T)$, medullary capillaries, and in collecting ducts $(c)$. Binding of $6 \mathrm{D} 1$ was weakly seen in the vascular bundles.

two membrane inhibitors, 5I2Ag (10) (rat Crry/p65 [11-13]) and rat CD59 $(16,17)$, are similar to those of human counterparts (28). Since the alternative complement pathway is under the pressure of automatic activation due to spontaneous hydrolysis of thioester bond on $\alpha$-chain of C3 molecule, C3b molecules can happen to be generated even on the self cell membranes.
These C3b molecules initiate amplification of alternative complement pathway unless membrane inhibitors such as rat Crry/ p65 restrict it. Our previous work testified to this notion using an in vivo model (15). In the present work, it was clearly demonstrated that functional suppression of rat Crry/p65 of a rat kidney by $5 \mathrm{I} 2$ (without $\mathrm{Fc}$ region) resulted in $\mathrm{C} 3$ and $\mathrm{C} 5 \mathrm{~b}-9$ 

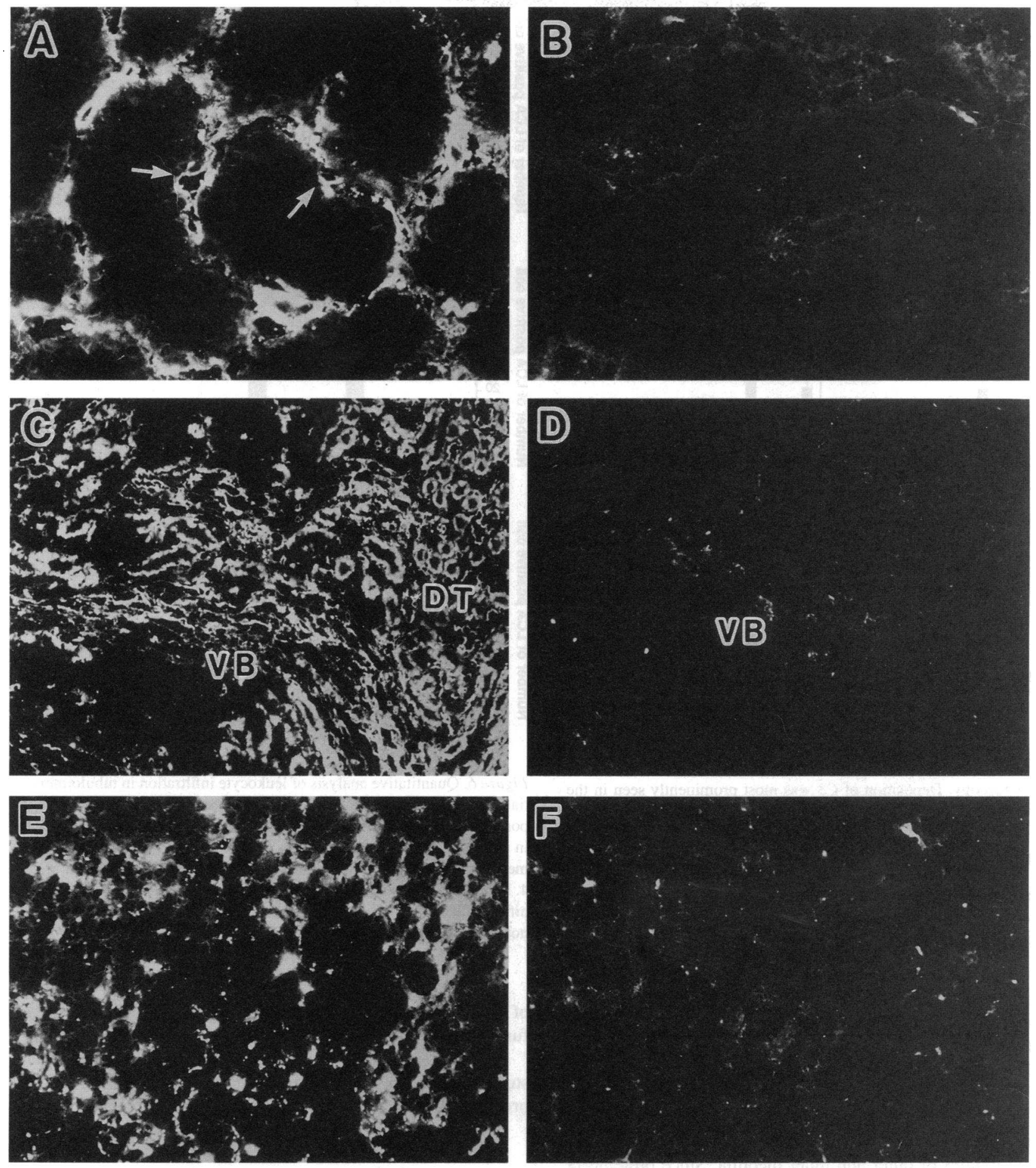

Figure 4. Deposition of rat $\mathrm{C} 3$ in the kidney $15 \mathrm{~min}$ after perfusion/recirculation. ( $A, C$, and $E)$ Group I: $(B, D$, and $F)$ group IV; $(\times 200) ;(A$ and $B$ ) cortex; $(C$ and $D)$ outer medulla; $(E$ and $F)$ inner medulla. Rat $C 3$ was strongly detected in the peritubular capillaries (arrows), vascular bundles $(V B)$, distal tubules $(D T)$, and in the inner medulla of a group I rat. In contrast, there was no significant binding of rat $C 3$ in the kidney from group IV.

deposition, tubular damage, and interstitial leukocyte infiltration within several days, whereas suppression of CD59 had no such effect.

By blocking the function of rat Crry/p65 using mAb 5I2, complement activation at $\mathrm{C} 3$ level was accelerated and formation of membrane attack complex was subsequently increased.
The increased amount of formed membrane attack complex might exceed the inhibitory activity of CD59. This is thought to be the reason why not only C3 but also C5b-9 was deposited in the kidney tissues of group I despite the presence of CD59. Once alternative pathway of complement was activated in the kidney, increased vascular permeability and direct action of 
$\mathrm{C}_{3}$
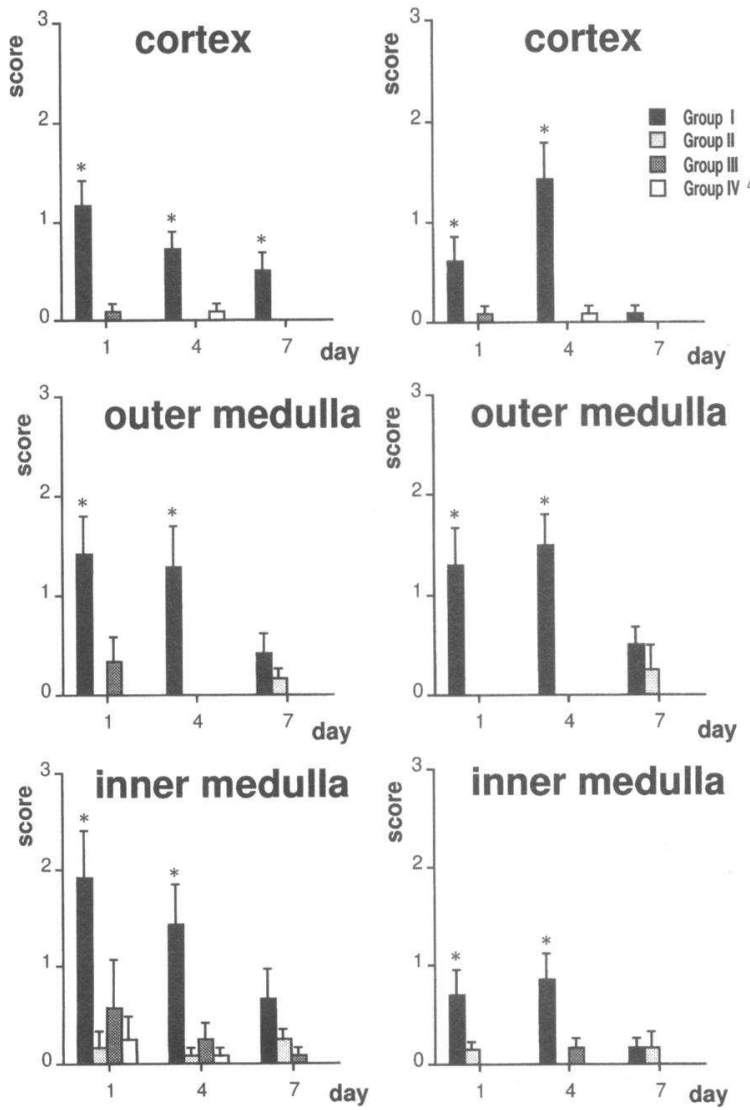

Figure 5. Semiquantitative analysis of deposition of rat C3 and C5b-9 (membrane attack complex) in the kidney assessed by immunofluorescence microscopy. Deposition of C3 was most prominently seen in the outer and inner medulla at days 1 and 4 in rats of group I. Deposition of C5b-9 was clearly seen in these rats in the outer medulla at day 1 and day 4. Complement deposition was decreased by day 7 . In other groups of rats (II, III, and IV), complement deposition was minimal throughout the experiments. Data are shown in mean (column) and standard error (bar). * Significant difference between group I and other groups; ${ }^{\Delta}$ group IV was not studied at day 7.

complement on tubulointerstitial tissues occurred as the first step of renal injury $(\sim 15 \mathrm{~min})$. At day 1 , cellular infiltration in the interstitial tissue was minimal while tubular degeneration, dilatation, and cast formation were already present. At this stage, there was significant deposition of $\mathrm{C} 3$ and C5b-9 along tubular basement membrane of cortex and in the vascular structures and tubules of outer and inner medulla. Since proteinuria was very mild, it is highly probable that most of the complement proteins derived directly from the circulation due to the increased vascular permeability. Anaphylatoxins, C5a and C3a, generated in the process of complement activation are considered as the responsible pathogenetic mediators. Events after the initial renal injury were the further development of tubular dilatation, cast formation, degeneration, and interstitial leukocyte infiltration. These might be the consequence of tubular dysfunction and various chemotactic mediators produced by kidney tissues and biologically active complement degradation products. At day 7, renal pathology tended to heal. Thus, single perfusion of $5 \mathrm{I} 2$ into the kidney and subsequent restoration
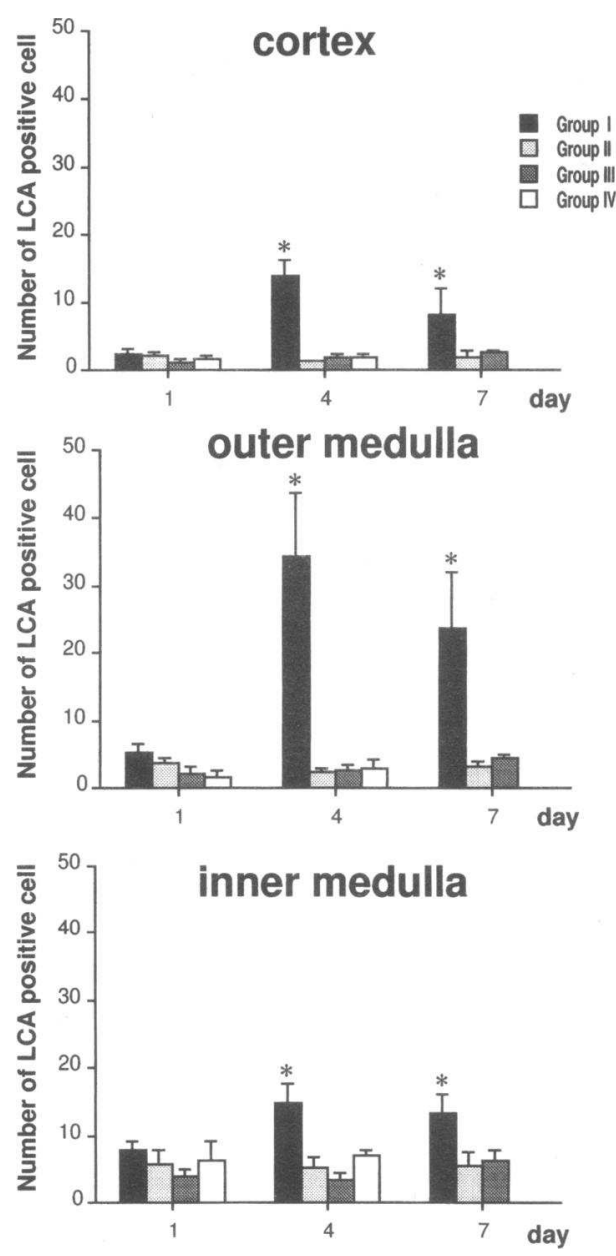

Figure 6. Quantitative analysis of leukocyte infiltration in tubulointerstitial tissue. Leukocyte infiltration was assessed by the number of LCApositive cells present in the section, and it was most prominently seen in the outer medulla of group I rats at days 4 and 7. In cortex and inner medulla, mild but significant leukocyte infiltration was observed at day 4. In other groups of rats, leukocyte infiltration was not observed. * Significant difference between group I and other groups; ${ }^{\Delta}$ group IV was not studied at day 7 .

of blood flow induced transient tubulointerstitial injury due to functional suppression of rat Crry/p65.

In the present study, kidney functions (GFR and RPF) were studied at day 4 when morphological alterations were most prominently seen in group I. There was no significant difference

Table II. Results of Functional Studies

\begin{tabular}{rccccc}
\hline Group & $n$ & RPF & Extraction & GFR & Filtration fraction \\
\hline & & $\mathrm{ml} / \mathrm{min}$ & & $\mathrm{m} / \mathrm{min}$ & \\
I & 6 & $5.44 \pm 0.26$ & $0.84 \pm 0.03$ & $1.20 \pm 0.07$ & $0.22 \pm 0.01$ \\
II & 6 & $6.08 \pm 0.37$ & $0.87 \pm 0.02$ & $1.31 \pm 0.08$ & $0.22 \pm 0.01$ \\
III & 6 & $6.18 \pm 0.34$ & $0.88 \pm 0.01$ & $1.53 \pm 0.13$ & $0.24 \pm 0.01$ \\
IV & 6 & $6.38 \pm 0.43$ & $0.87 \pm 0.12$ & $1.33 \pm 0.06$ & $0.20 \pm 0.01$
\end{tabular}

Functions of the left kidneys were studied at day 4 and there was no statistical difference among groups. 


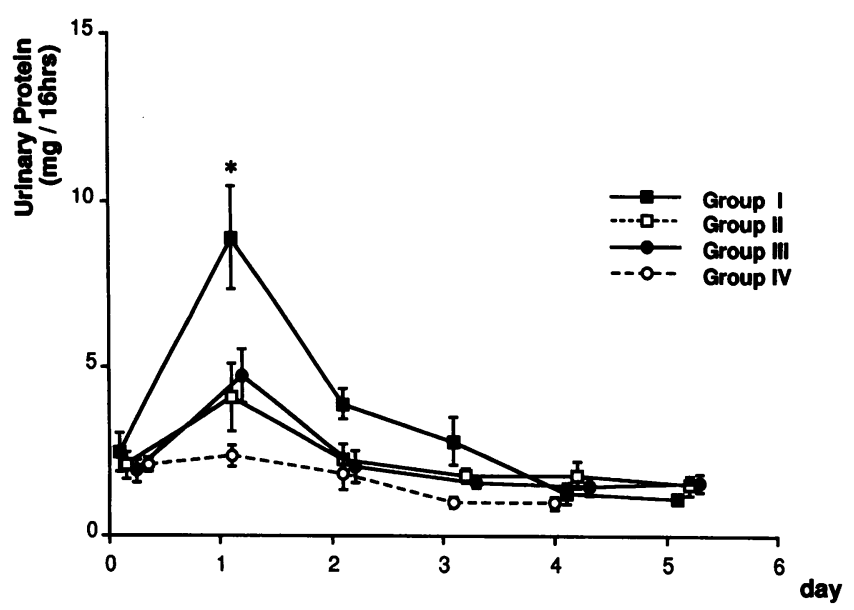

Figure 7. Urinary protein excretion. Mild proteinuria was observed at day 1 (the same day of perfusion recirculation) in rats of group I. Proteinuria was very transient and was not observed at day 2 and onwards. In other groups of rats, there was no significant proteinuria throughout the experiment.

between group I and other groups in these functional parameters. This implies that the pathological changes induced by single perfusion of 512 were not severe enough to affect GFR and RPF. Other sensitive parameters might be required for the detection of functional abnormalities in the present model.

In contrast to tubulointerstitial tissue, glomeruli did not show any significant change by $5 \mathrm{I} 2$ or $6 \mathrm{D} 1$, although significant binding of antibodies after perfusion with $5 \mathrm{I} 2$ and $6 \mathrm{D} 1$ was observed. Complement deposition in the glomerulus was transiently seen $15 \mathrm{~min}$ after perfusion/recirculation in rats perfused with 5I2, but it was no longer observed at day 1 and onwards. This may imply that glomerulus expresses more Crry/p65 than is neutralized by perfused $5 \mathrm{I} 2$ or that glomerulus has other complement regulator( $s$ ) which can compensate for the neutralized Crry/p65. It is, however, highly probable that suppression of rat Crry/p65 in the glomerulus may worsen the complementmediated injury when there is complement-activating stimuli. In fact, our recent study showed that suppression of glomerular Crry/p65 by $5 \mathrm{I} 2$ worsened anti-Thy 1 nephritis and supported this hypothesis (36). In an experimental glomerulonephritis using lectin and its antibodies, this is also true for rat CD59, the target molecule of 6D1 (22).

Results obtained in the present work support the ideas that the kidney is always under the threat of autologous complement attack and that membrane inhibitors of complement abundantly expressed in the kidney play a vital protective role against complement-mediated tissue injury in normal and diseased conditions. Complement-mediated renal injury will progress when the balance between activation and inhibition of complement is disregulated. The present work provides a new insight into the role of the membrane inhibitor of complement and has relevance to the understanding of the mechanism of tubulointerstitial injury in humans.

\section{Acknowledgments}

The authors thank N. Suzuki, M. Miyawaki, N. Kuno, and M. Hagino for their excellent technical assistance. Dr. N. Sakamoto (a director of Chubu Rousai Hospital and former chairman of the Third Department of Internal Medicine) gave the authors continuous encouragement and valuable suggestions.

This work was supported by research grants from Japan Ministry of Education, Science and Culture, and the 1994 research grant from The Aichi Kidney Foundation. B. P. Morgan is a Wellcome Senior Research Fellow.

\section{References}

1. Striker, G. E., L. I. Schainuck, R. E. Cutler, and E. P. Benditt. 1970 Structural-functional correlations in renal disease. I. A method for assaying and classifying histopathologic changes in renal disease. Hum. Pathol. 1:615-630.

2. Schainuck, L. I., G. E. Striker, R. E. Cutler, and E. P. Benditt. 1970. Structural-functional correlations in renal disease. II. The correlations. Hum. Pathol. 1:631-640.

3. Nath, K. A. 1992. Tubulointerstitial changes as a major determinant in the progression of renal disease. Am. J. Kidney Dis. 20:1-17.

4. Nath, K. A., M. K. Hostetter, and T. H. Hostetter. 1985. Pathophysiology of chronic tubulo-interstitial disease in rats. Interactions of dietary acid load, ammonia, and complement component C3. J. Clin. Invest. 76:667-675.

5. Tolins, J. P., M. K. Hostetter, and T. H. Hostetter. 1987. Hypokalemic nephropathy in the rat: role of ammonia in chronic tubular injury. J. Clin. Invest. 79:1447-1458.

6. Hostetter, M. K., and D. L. Gordon. 1987. Biochemistry of C3 and related thioester proteins in infection and inflammation. Rev. Infect. Dis. 9:97-109.

7. Hostetter, M. K. 1993. The third component of complement: new functions for an old friend. J. Lab. Clin. Med. 122:491-496.

8. Ichida, S., Y. Yuzawa, H. Okada, K. Yoshioka, and S. Matsuo. 1994. Localization of the complement regulatory proteins in the normal human kidney. Kidney Int. 46:89-96.

9. Takizawa, H., N. Okada, and H. Okada. 1994. Complement inhibitor of rat cell membrane resembling mouse Crry/p65. J. Immunol. 152:3032-3038.

10. Sakurada, C., H. Seno, N. Dohi, H. Takizawa, M. Nonaka, N. Okada, and H. Okada. 1994. Molecular cloning of the rat complement regulatory protein, 512 antigen. Biochem. Biophys. Res. Commun. 198:819-826.

11. Molina, H., W. Wong, T. Kinoshita, C. Brenner, S, Foley, and V. M. Holers. 1992. Distinct receptor and regulatory properties of recombinant mouse complement receptor 1 (CR1) and Crry, the two genetic homologues of human CR1. J. Exp. Med. 175:121-129.

12. Martin, B. K., and J. H. Weis. 1993. Functional identification of transcription control sequences of the mouse Crry gene. J. Immunol. 151:857-869.

13. Foley, S., B. Li, M. Dehoff, H. Molina, and V. M. Holers. 1993. Mouse Crry/p65 is a regulator of the alternative pathway of complement activation. Eur. J. Immunol. 23:1381-1384.

14. Kim, Y. U., T. Kinoshita, H. Molina, D. Hourcade, T. Seya, L. M. Wagner, and V. M. Holers. 1995. Mouse complement regulatory protein Crry/p65 uses the specific mechanisms of both human decay-accelerating factor and membrane cofactor protein. J. Exp. Med. 181:151-159.

15. Matsuo, S., S. Ichida, H. Takizawa, N. Okada, L. Baranyi, A. Iguchi, B. P. Morgan, and H. Okada. 1994. In vivo effects of monoclonal antibodies which functionally inhibit complement regulatory proteins in rats. J. Exp. Med. 180:1619-1627.

16. Hughes, T. R., S. J. Piddlesden, J. D. Williams, R. A. Harrison, and B. P. Morgan. 1992. Isolation and characterization of a membrane protein from rat erythrocytes which inhibits lysis by the membrane attack complex of rat complement. Biochem. J. 284: 169-176.

17. Rushmere, N. K., R. A. Harrison, C. W. van den Berg, and B. P. Morgan. 1994. Molecular cloning of the rat analogue of human CD59: structural comparison with human CD59 and identification of a putative active site. Biochem. J. 304:595-601.

18. Schulze, M., P. J. Baker, D. T. Perkinson, R. J. Johnson, R. F. Ochi, R. A. K. Stahl, and W. G. Couser. 1989. Increased urinary excretion of C5b-9 distinguishes passive Heymann nephritis. Kidney Int. 35:60-68.

19. Guesdon, J., T. Temynck, and S. Abrameas. 1979. The use of avidinbiotin interaction in immunoenzymatic techniques. J. Histochem. Cytochem. 27:1131-1139.

20. Watanabe, N., S. Kamei, A. Ohkubo, M. Yamanaka, S. Ohsawa, K. Makino, and K. Tokuda. 1986. Urinary protein as measured with a pyrogallol red-molybdate complex, manually and in a Hitachi 726 automated analyzer. Clin. Chem. 32:1551-1554.

21. Matsuo, S., F. Yoshida, Y. Yuzawa, S. Hara, A. Fukatsu, Y. Watanabe, and N. Sakamoto. 1989. Experimental glomerulonephritis induced in rats by a lectin and its antibodies. Kidney Int. 36: 1011-1021.

22. Matsuo, S., H. Nishikage, F. Yoshida, A. Nomura, S. J. Piddlesden, and B. P. Morgan. 1994. Role of CD59 in experimental glomerulonephritis in rats. Kidney Int. 46:191-200.

23. Gröne, H. J., K. Weber, E. Gröne, U. Helmchen, and M. Osborn. 1987. 
Coexpression of keratin and vimentin in damaged and regenerating tubular epithelia of the kidney. Am. J. Pathol. 129:1-8.

24. Eddy, A. A. 1991. Tubulointerstitial nephritis during the heterologous phase of nephrotoxic serum nephritis. Nephron. 59:304-313.

25. Platt, J., and A. F. Michael. 1983. Retardation of fading and enhancement of intensity of immunofluorescence by p-phenylene-diamine. J. Histochem. Cytochem. 31:840-842.

26. Dische, Z., and E. Borenfreund. 1951. A new spectrophotometric method for the detection and determination of keto sugars and trioses. J. Biol. Chem. 192:583-587.

27. Marshall, E. K., Jr. 1937. Determination of sulfanilamide in blood and urine. J. Biol. Chem. 122:263-273.

28. Funabashi, K., N. Okada, S. Matsuo, T. Yamamoto, B. P. Morgan, and H. Okada. 1994. Tissue distribution of complement regulatory membrane protein in rats. Immunology. 81:444-451.

29. Seya, T., J. Turner, and J. P. Atkinson. 1986. Purification and characterization of membrane cofactor protein (gp45-70) which is a cofactor for cleavage of C3b and C4b. J. Exp. Med. 163:837-855.

30. Nicholson-Weller, A., J. Burge, D. T. Fearon, P. F. Weller, and K. F. Austen. 1982. Isolation of a human erythrocyte membrane glycoprotein with decay-accelerating activity for $\mathrm{C} 3$ convertases of the complement system. $J$ Immunol. 129:184-189.
31. Zalman, L. S., L. M. Wood, and H. J. Müller-Eberhard. 1986. Isolation of a human erythrocyte membrane protein capable of inhibiting expression of homologous complement transmembrane channels. Proc. Natl. Acad. Sci. USA 83:6975-6979.

32. Holguin, M. H., L. R. Fredrick, N. J. Bernshaw, L. A. Wilcox, and C. J. Parker. 1989. Isolation and characterization of a membrane protein from norma human erythrocytes that inhibits reactive lysis of the erythrocytes of paroxysmal nocturnal hemoglobinuria. J. Clin. Invest. 84:7-17.

33. Sugita, Y., Y. Nakano, and M. Tomita. 1988. Isolation from human erythrocytes of a new membrane protein which inhibits the formation of complement transmembrane channels. J. Biochem. 104:633-637.

34. Okada, N., R. Harada, T. Fujita, and H. Okada. 1989. Monoclonal antibod ies capable of causing hemolysis of neuraminidase-treated human erythrocytes by homologous complement. J. Immunol. 143:2262-2266.

35. Davies, A., D. L. Simmons, G. Hale, R. A. Harrison, H. Tighe, P. J. Lachmann, and H. Waldmann. 1989. CD59, an Ly-6-like protein expressed in human lymphoid cells, regulates the action of the complement membrane attack complex on homologous cells. J. Exp. Med. 170:637-654.

36. Nishikage, H., L. Baranyi, H. Okada, N. Okada, K. Isobe, A. Nomura, F. Yoshida, and S. Matsuo. 1995. The role of a complement regulatory protein in rat mesangial glomerulonephritis. J. Am. Soc. Nephrol. 6:234-242. 